\title{
Estudo comparativo entre cloreto de mepiquat e cloreto de chlormequat aplicados no algodoeiro(1)
}

\author{
Fernando Mendes Lamas ${ }^{(2)}$
}

\begin{abstract}
Resumo - A comparação dos efeitos da aplicação dos reguladores de crescimento cloreto de mepiquat e cloreto de chlormequat isolados e combinados no algodoeiro (Gossypium hirsutum L.) cv. CNPA ITA 90 constituiu o objetivo do presente trabalho. O experimento foi conduzido no ano agrícola de 1998/99 no Município de Primavera do Leste, MT. Foram estudados 15 tratamentos com quatro repetições, utilizando-se o delineamento experimental de blocos casualizados. A altura das plantas com a aplicação parcelada foi $24 \%$ menor em relação à testemunha, independentemente do produto estudado. O peso do capulho foi maior com a aplicação de regulador de crescimento, e não dependeu do produto e do esquema de aplicação. O peso de 100 sementes depende da sequiência de aplicação e do produto utilizado; nos tratamentos em que as duas primeiras aplicações foram com cloreto de chlormequat, o peso de 100 sementes foi maior. Não houve efeito significativo dos reguladores de crescimento na porcentagem de fibras; entretanto, a produtividade de fibras foi influenciada pelo produto e pelo esquema de parcelamento; com três e duas aplicações do mesmo produto, a maior produtividade foi obtida com cloreto de mepiquat.
\end{abstract}

Termos para indexação: Gossypium hirsutum, substâncias de crescimento, fibra vegetal, rendimento.

\section{Comparative study of mepiquat chloride and chlormequat chloride application in cotton}

\begin{abstract}
This work was carried out to compare the effects of the growth regulators mepiquat chloride and chlormequat chloride applied in combination or separately in the cotton (Gossypium hirsutum L.) cultivar CNPA ITA 90. The experiment was evaluated in Mato Grosso State, Brazil, in the agricultural year of 1998/99. Fifteen treatments were studied with four replications, using randomized complete block design. The height of the plants with the parceled application was $24 \%$ smaller in relation to the control treatment, independently on the chemical. The boll weight was greater with the application of growth regulator, not depending on the product and the application outline. The weight of 100 seeds depends on the application sequence and on the chemical; it was larger when the first two applications were with chlormequat chloride. There was not significant effect of the growth regulators in the fiber percentage; however, the fiber productivity was influenced by the product and by the application outline; with three or two applications of the same product, the largest productivity was obtained with mepiquat chloride.
\end{abstract}

Index terms: Gossypium hirsutum, plant growth substances, plant fibres, yields.

\section{Introdução}

Os reguladores de crescimento são substâncias químicas sintéticas que alteram o balanço hormonal das plantas. O hormônio vegetal sobre o qual os reguladores de crescimento atuam é a giberelina, que tem a sua biossíntese inibida (Reddy et al., 1995).

\footnotetext{
(1) Aceito para publicação em 29 de novembro de 2000.

(2) Embrapa-Centro de Pesquisa Agropecuária do Oeste, Caixa Postal 661, CEP 79804-970 Dourados, MS.

E-mail: lamas@cpao.embrapa.br
}

Os principais efeitos dos reguladores de crescimento sobre o algodoeiro são: redução da altura, do tamanho dos ramos vegetativos e reprodutivos, do comprimento dos internódios, do número de folhas quando da colheita, aumento da precocidade, do peso dos capulhos e de 100 sementes (Cothren \& Oosterhuis, 1993; Pípolo et al., 1993; Carvalho et al., 1994; Lamas, 1997; Athayde \& Lamas, 1999).

Quando o algodoeiro é cultivado com adequada disponibilidade de água, de nutrientes e condições 
climáticas favoráveis, verifica-se crescimento excessivo da parte vegetativa, o que pode interferir negativamente na produção. Nestas condições, o uso de substâncias reguladoras de crescimento torna-se indispensável (Reddy et al., 1992).

$\mathrm{O}$ equilíbrio entre as partes vegetativas e reprodutivas propicia um maior deslocamento de metabólitos para os frutos (Beltrão, 1996?).

Uma das estratégias agronômicas para o aumento da produtividade do algodoeiro é o manejo das plantas com reguladores de crescimento (Hodges et al., 1991).

No Brasil, encontram-se três produtos comerciais que são utilizados como reguladores de crescimento no algodoeiro, os quais têm como ingredientes ativos: cloreto de chlormequat, cloreto de clorocolina e cloreto de mepiquat. Estes produtos têm mecanismos e modo de ação semelhantes, ou seja, interferem na biossíntese do ácido giberélico, inibindo-a, o que resulta em redução do crescimento e alterações fisiológicas (Reddy et al., 1995; Marur, 1998).

O objetivo deste trabalho foi comparar os efeitos dos produtos cloreto de mepiquat e cloreto de chlormequat aplicados, isolados e combinados, sobre algumas características do algodoeiro.

\section{Material e Métodos}

O experimento foi conduzido no ano agrícola de (1998/99), em Primavera do Leste, MT. Utilizou-se a cultivar CNPA ITA 90, semeada em 20/12/98, com emergência em 26/12/98, no espaçamento entre fileiras de $0,90 \mathrm{~m}$ e densidade de 10 plantas $\mathrm{m}^{-1}$.

A adubação na semeadura foi de $24,0,72,0$ e $48,0 \mathrm{~kg} \mathrm{ha}^{-1}$ de N, $\mathrm{P}_{2} \mathrm{O}_{5}$ e $\mathrm{K}_{2} \mathrm{O}$, respectivamente.

A primeira adubação em cobertura foi realizada aos 24 dias após a emergência (DAE), com 30,0 + 30,0 + $0,75 \mathrm{~kg} \mathrm{ha}^{-1} \mathrm{de} \mathrm{N}, \mathrm{K}_{2} \mathrm{O}$ e B, respectivamente; e, a segunda, aos 45 DAE, com 40,0 + 40,0 + 1,0 $\mathrm{kg} \mathrm{ha}^{-1}$ de N, $\mathrm{K}_{2} \mathrm{O}$ e $\mathrm{B}$, respectivamente.
O delineamento experimental utilizado foi o de blocos casualizados, com quatro repetições. As parcelas experimentais foram compostas por seis fileiras com 5,0 m de comprimento. Como área útil foram consideradas as duas fileiras centrais $(1,8 \times 5,0 \mathrm{~m})$.

As doses de cloreto de mepiquat (CM) e de cloreto de chlormequat (CC), e as formas de intercalação dos produtos encontram-se na Tabela 1.

A aplicação dos reguladores de crescimento foi iniciada aos 45 DAE, quando as plantas apresentavam altura média de $0,52 \mathrm{~m}$, a segunda e a terceira aplicação foi aos 61 e 83 DAE, respectivamente.

A Tabela 2 apresenta os dados observados de precipitação pluvial $(\mathrm{mm})$, por decêndio, da área experimental.

Os reguladores de crescimento foram aplicados no período da manhã, antes das $9 \mathrm{~h}$, utilizando-se pulverizador de alta precisão, a $40 \mathrm{lb} \mathrm{pol}^{-2}$, com bico D 14 e vazão de $200 \mathrm{~L}_{\text {de calda ha }}{ }^{-1}$.

Antes da primeira colheita, em cada parcela experimental, dentro da área útil, foi medida a altura de cinco plantas tomadas ao acaso.

Tabela 1. Doses de cloreto de mepiquat (CM) e de cloreto de chlormequat (CC) e formas de intercalação estudadas. Dourados, MS, 1999.

\begin{tabular}{lccc}
\hline \multirow{2}{*}{ Tratamento } & \multicolumn{3}{c}{ Doses dos produtos $\left(\mathrm{g} \mathrm{ha}^{-1}\right)$} \\
\cline { 2 - 4 } & $1^{\mathrm{a}}$ aplicação & $2^{\underline{\mathrm{a}} \text { aplicação }}$ & $3^{\mathrm{a}}$ aplicação \\
\hline T1 & 0,0 & 0,0 & 0,0 \\
T2 & $25,0(\mathrm{CC})$ & $35,0(\mathrm{CC})$ & $40,0(\mathrm{CC})$ \\
T3 & $25,0(\mathrm{CC})$ & $35,0(\mathrm{CC})$ & $20,0(\mathrm{CM})$ \\
T4 & $25,0(\mathrm{CC})$ & $17,5(\mathrm{CM})$ & $40,0(\mathrm{CC})$ \\
T5 & $12,5(\mathrm{CM})$ & $35,0(\mathrm{CC})$ & $40,0(\mathrm{CC})$ \\
T6 & $12,5(\mathrm{CM})$ & $17,5(\mathrm{CM})$ & $40,0(\mathrm{CC})$ \\
T7 & $25,0(\mathrm{CC})$ & $17,5(\mathrm{CM})$ & $20,0(\mathrm{CM})$ \\
T8 & $12,5(\mathrm{CM})$ & $17,5(\mathrm{CM})$ & $20,0(\mathrm{CM})$ \\
T9 & $12,5(\mathrm{CM})$ & $35,0(\mathrm{CC})$ & $20,0(\mathrm{CM})$ \\
T10 & 0,0 & $50,0(\mathrm{CC})$ & $50,0(\mathrm{CC})$ \\
T11 & 0,0 & $25,0(\mathrm{CM})$ & $50,0(\mathrm{CC})$ \\
T12 & 0,0 & $50,0(\mathrm{CC})$ & $25,0(\mathrm{CM})$ \\
T13 & 0,0 & $25,0(\mathrm{CM})$ & $25,0(\mathrm{CM})$ \\
T14 & 0,0 & 0,0 & $100,0(\mathrm{CC})$ \\
T15 & 0,0 & 0,0 & $50,0(\mathrm{CM})$ \\
\hline
\end{tabular}

Tabela 2. Precipitação pluvial (mm), por decêndio, durante os meses de condução do experimento.

\begin{tabular}{lcccccc}
\hline Decêndio & Dezembro & Janeiro & Fevereiro & Março & Abril & Maio \\
\hline 1 & 312,00 & 74,00 & 25,00 & 257,00 & 23,00 & 38,00 \\
2 & 180,00 & 141,00 & 30,00 & 138,00 & 35,00 & 0,00 \\
3 & 124,00 & 155,00 & 221,00 & 145,00 & 82,50 & 0,00 \\
\hline Total & 616,00 & 370,00 & 276,00 & 540,00 & 140,50 & 38,00 \\
\hline
\end{tabular}


Na primeira colheita, realizada aos 157 DAE, foram coletados 20 capulhos do terço médio das plantas, sendo um por planta, para as seguintes determinações: peso do capulho e de 100 sementes, e porcentagem de fibra. A fibra obtida de cada amostra, após o descaroçamento, foi analisada no laboratório de Tecnologia de Fibras da EmbrapaCentro Nacional de Pesquisa de Algodão, determinandose comprimento, uniformidade de comprimento, índice Micronaire e resistência. A precocidade foi calculada considerando-se a produção obtida na primeira colheita em relação à produção total.

Os resultados obtidos foram submetidos a análise de variância com o desdobramento dos graus de liberdade dos tratamentos, com o objetivo de se obter informações mais específicas relacionadas com o comportamento de cada um dos componentes do desdobramento, de acordo com o método proposto por Banzatto \& Kronka (1992). Os contrastes estabelecidos para comparação dos diversos tratamentos (T) são os seguintes:

$$
\begin{aligned}
& \mathrm{Y} 1= 14 \mathrm{~T} 1-\mathrm{T} 2-\mathrm{T} 3-\mathrm{T} 4-\mathrm{T} 5-\mathrm{T} 6-\mathrm{T} 7-\mathrm{T} 8-\mathrm{T} 9- \\
& \mathrm{T} 10-\mathrm{T} 11-\mathrm{T} 12-\mathrm{T} 13-\mathrm{T} 14-\mathrm{T} 15 \\
& \mathrm{Y} 2= \mathrm{T} 14-\mathrm{T} 15 \\
& \mathrm{Y} 3= \mathrm{T} 10-\mathrm{T} 13 \\
& \mathrm{Y} 4= \mathrm{T} 11-\mathrm{T} 12 \\
& \mathrm{Y} 5=\mathrm{T} 2-\mathrm{T} 8 \\
& \mathrm{Y} 6=\mathrm{T} 2-\mathrm{T} 3 \\
& \mathrm{Y} 7=\mathrm{T} 3-\mathrm{T} 4 \\
& \mathrm{Y} 8=\mathrm{T} 5-\mathrm{T} 6 \\
& \mathrm{Y} 9=\mathrm{T} 7-\mathrm{T} 8 \\
& \mathrm{Y} 10=\mathrm{T} 8-\mathrm{T} 9 \\
& \mathrm{Y} 11=\mathrm{T} 10-\mathrm{T} 11 \\
& \mathrm{Y} 12=2 \mathrm{~T} 2-\mathrm{T} 10-\mathrm{T} 14 \\
& \mathrm{Y} 13= \\
& \mathrm{Y} 14=-\mathrm{T} 2-\mathrm{T} 3-\mathrm{T} 4+\mathrm{T} 13-\mathrm{T} 15 .
\end{aligned}
$$

\section{Resultados e Discussão}

A altura das plantas foi significativamente reduzida com a aplicação do regulador de crescimento (Tabela 3). Tanto com o cloreto chlormequat (Y12) como com o cloreto de mepiquat (Y14), o efeito do parcelamento na redução da altura das plantas foi significativamente maior, não diferindo entre eles (Tabelas 3 e 4). Os resultados foram semelhantes aos obtidos por Laca-Buendia (1989), Wallace et al. (1993) e Furlani Júnior et al. (1999), que estudaram o cloreto de mepiquat. Com duas aplicações (Y3), o efeito do cloreto de chlormequat (T10) sobre a redução da altura das plantas foi significativamente mai- or em comparação ao cloreto de mepiquat (T13). Não se verificou diferença significativa entre os produtos quando estes foram aplicados em uma única vez (Tabelas 3, 4 e 5). De acordo com os resultados obtidos, fica evidente que a aplicação parcelada de regulador de crescimento proporciona maior redução na altura das plantas, independentemente do produto utilizado.

O peso do capulho foi significativamente maior $(\mathrm{P}<0,01)$ nos tratamentos que receberam reguladores de crescimento (Y1), independentemente da forma em que foram aplicados (Tabelas 3, 4 e 5). Este comportamento deve-se a um maior equilíbrio entre as partes vegetativas e reprodutivas, possibilitando aos frutos um maior aproveitamento dos fotoassimilados (Beltrão, 1996). Resultados semelhantes foram obtidos por Carvalho et al. (1994) e McCarty \& Hedin (1994).

Quando foram feitas três aplicações (Y7), o tratamento em que as duas primeiras foram com cloreto de chlormequat (T3), o peso de 100 sementes foi significativamente maior quando comparado com o tratamento onde a segunda aplicação foi com cloreto de mepiquat (T4). Quando foram feitas duas aplicações (Y3), o efeito sobre o peso de 100 sementes diferiu significativamente entre os produtos, e o tratamento com cloreto de chlormequat (T12) proporcionou maior peso de 100 sementes (Tabelas 3 e 4). Lamas (1997) verificou aumento do peso de 100 sementes com a aplicação de cloreto de mepiquat, e o mesmo foi verificado por Carvalho et al. (1994), quando compararam o efeito dos dois produtos sobre o peso de 100 sementes.

A aplicação de regulador de crescimento não teve efeito significativo sobre a porcentagem de fibras $(\mathrm{P}>0,05)$ (Tabela 3). Resultados semelhantes foram obtidos por Lamas (1997) e Athayde \& Lamas (1999) em trabalhos desenvolvidos com cloreto de mepiquat. Entretanto, Carvalho et al. (1994), comparando o efeito de diversos produtos reguladores de crescimento, constataram redução na porcentagem de fibras com a aplicação de cloreto de chlormequat. Pelos resultados obtidos, pode-se inferir que os efeitos dos reguladores de crescimento sobre os principais componentes do peso do capulho (sementes + fibra) são diferentes.

Pela Tabela 3 verifica-se que o efeito dos reguladores de crescimento sobre a produtividade de fibras não foi significativo (Y1); entretanto, verificaram-se diferenças entre os reguladores (Y3 e Y5).

Nos tratamentos em que foram feitas duas aplicações com o mesmo produto (T10 e T13), a média de 
Tabela 3. Quadrados médios obtidos nas análises de variância da altura das plantas, peso do capulho, peso de 100 sementes, porcentagem e produção de fibras e precocidade.

\begin{tabular}{|c|c|c|c|c|c|c|}
\hline Contraste & $\begin{array}{l}\text { Altura das } \\
\text { plantas }\end{array}$ & $\begin{array}{l}\text { Peso do } \\
\text { capulho }\end{array}$ & $\begin{array}{l}\text { Peso de } 100 \\
\text { sementes }\end{array}$ & $\begin{array}{l}\text { Porcentagem } \\
\text { de fibras }\end{array}$ & $\begin{array}{l}\text { Produção de } \\
\text { fibras }\end{array}$ & Precocidade \\
\hline $\mathrm{Y1}$ & $1.804,78^{* *}$ & $0,56 * *$ & $0,57^{\mathrm{ns}}$ & $0,59^{\mathrm{ns}}$ & $8.314,44^{\mathrm{ns}}$ & $25,08^{\mathrm{ns}}$ \\
\hline $\mathrm{Y} 2$ & $58,64^{\mathrm{ns}}$ & $0,09^{\mathrm{ns}}$ & $0,08^{\mathrm{ns}}$ & $0,32^{\mathrm{ns}}$ & $1.499,71^{\mathrm{ns}}$ & $1,32^{\mathrm{ns}}$ \\
\hline Y3 & $1.127,89 * *$ & $0,04^{\mathrm{ns}}$ & $0,72 *$ & $0,12^{\text {ns }}$ & $191.592,26 *$ & $52,38 *$ \\
\hline Y4 & $156,03^{\mathrm{ns}}$ & $0,23^{\mathrm{ns}}$ & $0,06^{\mathrm{ns}}$ & $0,00^{\mathrm{ns}}$ & $5.709,96^{\mathrm{ns}}$ & $0,21^{\mathrm{ns}}$ \\
\hline Y5 & $260,69^{\mathrm{ns}}$ & $0,02^{\mathrm{ns}}$ & $0,05^{\mathrm{ns}}$ & $0,28^{\mathrm{ns}}$ & $164.005,53^{*}$ & $31,49^{\mathrm{ns}}$ \\
\hline Y6 & $5,03^{\mathrm{ns}}$ & $0,12^{\mathrm{ns}}$ & $0,00^{\mathrm{ns}}$ & $0,06^{\mathrm{ns}}$ & $7.849,79^{\text {ns }}$ & $1,76^{\mathrm{ns}}$ \\
\hline Y7 & $86,59^{\text {ns }}$ & $0,13^{\mathrm{ns}}$ & $0,84 *$ & $1,28^{\mathrm{ns}}$ & $51.688,68^{\mathrm{ns}}$ & $6,31^{\mathrm{ns}}$ \\
\hline Y8 & $107,53^{\mathrm{ns}}$ & $0,01^{\mathrm{ns}}$ & $0,01^{\mathrm{ns}}$ & $0,00^{\mathrm{ns}}$ & $4.602,15^{\mathrm{ns}}$ & $15,27^{\mathrm{ns}}$ \\
\hline Y9 & $3,55^{\mathrm{ns}}$ & $0,06^{\mathrm{ns}}$ & $0,25^{\mathrm{ns}}$ & $0,03^{\mathrm{ns}}$ & $1.145,48^{\mathrm{ns}}$ & $31,95^{\text {ns }}$ \\
\hline Y10 & $29,36^{\mathrm{ns}}$ & $0,03^{\mathrm{ns}}$ & $0,21^{\mathrm{ns}}$ & $0,78^{\mathrm{ns}}$ & $45.474,95^{\mathrm{ns}}$ & $13,35^{\text {ns }}$ \\
\hline Y11 & $346,63^{\mathrm{ns}}$ & $0,29^{\mathrm{ns}}$ & $0,24^{\mathrm{ns}}$ & $0,10^{\mathrm{ns}}$ & $71.098,25^{\mathrm{ns}}$ & $3,55^{\mathrm{ns}}$ \\
\hline Y12 & $1.014,03 * *$ & $0,01^{\mathrm{ns}}$ & $0,11^{\mathrm{ns}}$ & $0,01^{\mathrm{ns}}$ & $54.988,94^{\mathrm{ns}}$ & $6,89^{\mathrm{ns}}$ \\
\hline Y13 & $105,19^{\mathrm{ns}}$ & $0,08^{\mathrm{ns}}$ & $0,36^{\mathrm{ns}}$ & $0,30^{\mathrm{ns}}$ & $430,55^{\mathrm{ns}}$ & $32,20^{\mathrm{ns}}$ \\
\hline Y14 & $793,45 *$ & $0,05^{\mathrm{ns}}$ & $0,54^{\mathrm{ns}}$ & $0,28^{\mathrm{ns}}$ & $7,69^{\mathrm{ns}}$ & $0,12^{\text {ns }}$ \\
\hline Tratamentos & $515,17 * *$ & 0,14 & $0,37 *$ & $0,32^{\mathrm{ns}}$ & $46.350,19^{\mathrm{ns}}$ & $13,12^{\text {ns }}$ \\
\hline Blocos & 248,50 & 0,09 & 0,03 & 1,11 & $19.784,00$ & 10,83 \\
\hline Resíduo & 139,70 & 0,07 & 0,17 & 0,53 & $34.912,89$ & 10,60 \\
\hline C.V. (\%) & 9,08 & 4,34 & 4,10 & 1.68 & 8.85 & 5,28 \\
\hline
\end{tabular}

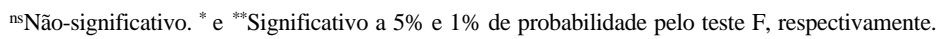

Tabela 4. Médias da altura das plantas, peso do capulho, peso de 100 sementes e porcentagem de fibras em cada comparação estabelecida.

\begin{tabular}{lcccc}
\hline Contraste & Altura das plantas (m) & Peso do capulho (g) & Peso de 100 sementes (g) & $\begin{array}{c}\text { Porcentagem } \\
\text { de fibras }(\%)\end{array}$ \\
\hline Y1 & $1,51-1,28^{* *}$ & $5,82-6,21^{* *}$ & $9,67-10,06^{\mathrm{ns}}$ & $43,72-43,36^{\mathrm{ns}}$ \\
Y2 & $1,47-1,41^{\mathrm{ns}}$ & $5,87-6,08^{\mathrm{ns}}$ & $9,75-9,55^{\mathrm{ns}}$ & $43,08-43,48^{\mathrm{ns}}$ \\
Y3 & $1,22-1,46^{* *}$ & $6,35-6,22^{\mathrm{ns}}$ & $10,45-9,85^{*}$ & $43,75-43,02^{\mathrm{ns}}$ \\
Y4 & $1,35-1,27^{\mathrm{ns}}$ & $5,97-6,31^{\mathrm{ns}}$ & $10,10-10,27^{\mathrm{ns}}$ & $43,50-43,50^{\mathrm{ns}}$ \\
Y5 & $1,15-1,27^{\mathrm{ns}}$ & $6,18-6,28^{\mathrm{ns}}$ & $10,30-10,15^{\mathrm{ns}}$ & $43,20-43,57^{\mathrm{ns}}$ \\
Y6 & $1,15-1,16^{\mathrm{ns}}$ & $6,18-6,42^{\mathrm{ns}}$ & $10,30-10,30^{\mathrm{ns}}$ & $43,20-43,02^{\mathrm{ns}}$ \\
Y7 & $1,16-1,23^{\mathrm{ns}}$ & $6,42-6,16^{\mathrm{ns}}$ & $10,30-9,65^{*}$ & $43,02-43,85^{\mathrm{ns}}$ \\
Y8 & $1,20-1,28^{\mathrm{ns}}$ & $6,31-6,27^{\mathrm{ns}}$ & $10,18-10,10^{\mathrm{ns}}$ & $43,22-43,23^{\mathrm{ns}}$ \\
Y9 & $1,28-1,27^{\mathrm{ns}}$ & $6,10-6,28^{\mathrm{ns}}$ & $9,80-10,15^{\mathrm{ns}}$ & $43,70-43,57^{\mathrm{ns}}$ \\
Y10 & $1,27-1,23^{\mathrm{ns}}$ & $6,28-6,40^{\mathrm{ns}}$ & $10,15-10,48^{\mathrm{ns}}$ & $43,57-42,95^{\mathrm{ns}}$ \\
Y11 & $1,22-1,35^{\mathrm{ns}}$ & $6,35-5,97^{\mathrm{ns}}$ & $10,45-10,10^{\mathrm{ns}}$ & $43,75-43,50^{\mathrm{ns}}$ \\
Y12 & $1,15-1,35^{* *}$ & $6,18-6,11^{\mathrm{ns}}$ & $10,30-10,10^{\mathrm{ns}}$ & $43,20-43,42^{\mathrm{ns}}$ \\
Y13 & $1,24-1,20^{\mathrm{ns}}$ & $6,32-6,21^{\mathrm{ns}}$ & $10,23-10,01^{\mathrm{ns}}$ & $43,24-43,44^{\mathrm{ns}}$ \\
Y14 & $1,27-1,44^{*}$ & $6,28-6,15^{\mathrm{ns}}$ & $10,15-9,70^{\mathrm{ns}}$ & $43,57-43,25^{\mathrm{ns}}$ \\
\hline
\end{tabular}

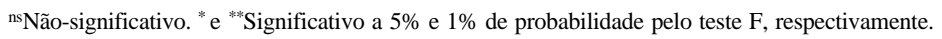

produtividade de fibras $\left(\mathrm{kg} \mathrm{ha}^{-1}\right)$ foi significativamente superior no tratamento com cloreto de mepiquat (T13), verificando-se o mesmo quando foram feitas três aplicações (Tabelas 3, 5 e 6). Quando os produtos foram aplicados de uma única vez, não se verifi- cou diferença significativa entre eles (Tabela 3 ). O efeito dos tratamentos sobre os componentes da produção avaliados (peso do capulho, peso de 100 sementes e porcentagem de fibra) não permite uma explicação consistente destes resultados, por causa 
Tabela 5. Médias da altura das plantas, peso do capulho, peso de 100 sementes, porcentagem e produtividade de fibras e precocidade.

\begin{tabular}{lcccccc}
\hline Tratamento & $\begin{array}{c}\text { Altura das } \\
\text { plantas }(\mathrm{m})\end{array}$ & $\begin{array}{c}\text { Peso do } \\
\text { capulho }(\mathrm{g})\end{array}$ & $\begin{array}{c}\text { Peso de 100 } \\
\text { sementes }(\mathrm{g})\end{array}$ & $\begin{array}{c}\text { Porcentagem } \\
\text { de fibras }(\%)\end{array}$ & $\begin{array}{c}\text { Produtividade de } \\
\text { fibras }\left(\mathrm{kg} \mathrm{ha}^{-1}\right)\end{array}$ & $\begin{array}{c}\text { Precocidade } \\
(\%)\end{array}$ \\
\hline T1 & 1,51 & 5,82 & 9,67 & 43,72 & $2.068,26$ & 73,86 \\
T2 & 1,15 & 6,18 & 10,30 & 43,20 & $1.951,80$ & 79,94 \\
T3 & 1,16 & 6,42 & 10,30 & 43,02 & $2.014,45$ & 78,61 \\
T4 & 1,23 & 6,16 & 9,65 & 43,85 & $2.175,21$ & 81,09 \\
T5 & 1,20 & 6,31 & 10,18 & 43,22 & $1.976,62$ & 79,95 \\
T6 & 1,28 & 6,27 & 10,10 & 43,23 & $2.024,39$ & 75,96 \\
T7 & 1,28 & 6,10 & 9,80 & 43,70 & $2.214,23$ & 79,98 \\
T8 & 1,27 & 6,28 & 10,15 & 43,57 & $2.238,16$ & 74,12 \\
T9 & 1,23 & 6,40 & 10,48 & 42,95 & $2.087,38$ & 77,96 \\
T10 & 1,22 & 6,35 & 10,45 & 43,75 & $1.973,48$ & 80,09 \\
T11 & 1,35 & 5,97 & 10,10 & 43,50 & $2.162,02$ & 78,21 \\
T12 & 1,27 & 6,31 & 10,27 & 43,50 & $2.108,59$ & 77,73 \\
T13 & 1,46 & 6,22 & 9,85 & 43,02 & $2.282,98$ & 72,52 \\
T14 & 1,47 & 5,87 & 9,75 & 43,08 & $2.217,33$ & 75,09 \\
T15 & 1,41 & 6,08 & 9,55 & 43,48 & $2.189,95$ & 76,31 \\
\hline
\end{tabular}

Tabela 6. Médias de produtividade de fibras e de precocidade em cada comparação estabelecida.

\begin{tabular}{lll}
\hline Contraste & $\begin{array}{c}\text { Produtividade de fibras } \\
\left(\mathrm{kg} \mathrm{ha}^{-1}\right)\end{array}$ & Precocidade $(\%)$ \\
\hline Y1 & $2.068,26-2.115,47^{\mathrm{ns}}$ & $73,86-77,68^{\mathrm{ns}}$ \\
Y2 & $2.217,33-2.189,95^{\mathrm{ns}}$ & $75,09-76,31^{\mathrm{ns}}$ \\
Y3 & $1.973,48-2.282,98^{*}$ & $80,09-72,52^{*}$ \\
Y4 & $2.162,02-2.108,59^{\mathrm{ns}}$ & $78,21-77,73^{\mathrm{ns}}$ \\
Y5 & $1.951,80-2.238,16^{*}$ & $79,94-74,12^{\mathrm{ns}}$ \\
Y6 & $1.951,80-2.014,45^{\mathrm{ns}}$ & $79,94-78,61^{\mathrm{ns}}$ \\
Y7 & $2.014,45-2.175,21^{\mathrm{ns}}$ & $78,61-81,09^{\mathrm{ns}}$ \\
Y8 & $1.976,62-2.024,39^{\mathrm{ns}}$ & $79,95-75,96^{\mathrm{ns}}$ \\
Y9 & $2.214,23-2.238,16^{\mathrm{ns}}$ & $79,98-74,12^{\mathrm{ns}}$ \\
Y10 & $2.238,16-2.087,38^{\mathrm{ns}}$ & $74,12-77,96^{\mathrm{ns}}$ \\
Y11 & $1.973,48-2.162,02^{\mathrm{ns}}$ & $80,09-78,21^{\mathrm{ns}}$ \\
Y12 & $1.951,80-2.095,41^{\mathrm{ns}}$ & $79,94-77,59^{\mathrm{ns}}$ \\
Y13 & $2.081,64-2.088,92^{\mathrm{ns}}$ & $79,99-79,91^{\mathrm{ns}}$ \\
Y14 & $2.238,16-2.236,46^{\mathrm{ns}}$ & $74,12-74,41^{\mathrm{ns}}$ \\
\hline
\end{tabular}

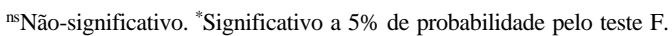

das diferenças de efeitos. Alguns autores obtiveram resultados semelhantes e outros contrários aos obtidos neste trabalho (Reddy et al., 1992; Wallace et al., 1993; Carvalho et al., 1994; Athayde \& Lamas, 1999). O efeito dos reguladores de crescimento sobre a produtividade de fibras depende da altura das plantas. Quando estas apresentam vegetação exube- rante, o efeito é significativo, graças ao melhor equilíbrio entre as partes vegetativas e reprodutivas, proporcionado pelo regulador de crescimento.

A precocidade, considerada como a porcentagem colhida na primeira colheita em relação à produção total, não foi significativamente influenciada pela aplicação dos reguladores de crescimento, verificando-se diferenças significativas entre os produtos em razão do número de aplicações (Tabela 3). Com duas aplicações de cloreto de chlormequat (T10), a precocidade foi significativamente maior do que com duas aplicações de cloreto de mepiquat (T13) (Tabelas 3, 5 e 6); esse resultado foi semelhante ao obtido quanto à produtividade de fibras. Lamas \& Staut (1999), avaliando o efeito de diferentes doses de cloreto de mepiquat, verificaram aumento da precocidade com o aumento da dose do produto. Pípolo et al. (1993) também encontraram efeito significativo sobre a precocidade, que aumentou com a aplicação de cloreto de clorocolina. Por ser a precocidade uma característica influenciada por vários fatores ambientais, é possível que um ou mais destes tenham interferido no efeito dos reguladores de crescimento sobre esta característica.

Nenhuma das características tecnológicas da fibra analisadas foram significativamente afetadas pela aplicação dos reguladores de crescimento, verifican- 
do-se efeitos significativos apenas do esquema de aplicação (Tabela 7).

A média do comprimento da fibra ( $\mathrm{mm}$ ) do tratamento em que as duas primeiras aplicações foram com cloreto de chlormequat (T3) foi significativamente maior do que a do tratamento onde a primeira $\mathrm{e}$ a terceira aplicação foi com cloreto de chlormequat (T4) (Tabelas 7, 8 e 9). No tratamento em que foram feitas duas aplicações com cloreto de mepiquat (T13), o valor do índice Micronaire foi significativamente menor quando comparado com o tratamento com duas aplicações de cloreto de chlormequat (T10). Em ambos

Tabela 7. Quadrados médios obtidos nas análises de variância de comprimento, uniformidade, resistência e micronaire da fibra.

\begin{tabular}{lcccc}
\hline Contraste & Comprimento & Uniformidade & Resistência & Micronaire \\
\hline Y1 & $0,01^{\text {ns }}$ & $0,82^{\text {ns }}$ & $0,34^{\text {ns }}$ & $0,09^{\text {ns }}$ \\
Y2 & $0,32^{\text {ns }}$ & $0,18^{\text {ns }}$ & $4,80^{\text {ns }}$ & $0,00^{\text {ns }}$ \\
Y3 & $0,06^{\text {ns }}$ & $0,45^{\text {ns }}$ & $0,50^{\text {ns }}$ & $0,21^{*}$ \\
Y4 & $0,15^{\mathrm{ns}}$ & $0,21^{\text {ns }}$ & $0,00^{\text {ns }}$ & $0,06^{\text {ns }}$ \\
Y5 & $0,21^{\text {ns }}$ & $0,91^{\text {ns }}$ & $0,55^{\text {ns }}$ & $0,02^{\text {ns }}$ \\
Y6 & $1,44^{\text {ns }}$ & $0,02^{\text {ns }}$ & $3,38^{\text {ns }}$ & $0,00^{\text {ns }}$ \\
Y7 & $4,50^{*}$ & $0,72^{\text {ns }}$ & $1,20^{\text {ns }}$ & $0,20^{\text {ns }}$ \\
Y8 & $0,13^{\text {ns }}$ & $0,04^{\text {ns }}$ & $1,20^{\text {ns }}$ & $0,01^{\text {ns }}$ \\
Y9 & $0,00^{\text {ns }}$ & $0,84^{\text {ns }}$ & $2,88^{\text {ns }}$ & $0,00^{\text {ns }}$ \\
Y10 & $4,06^{\text {ns }}$ & $0,84^{\text {ns }}$ & $0,00^{\text {ns }}$ & $0,03^{\text {ns }}$ \\
Y11 & $0,25^{\text {ns }}$ & $0,91^{\text {ns }}$ & $7,80^{\text {ns }}$ & $0,00^{\text {ns }}$ \\
Y12 & $0,17^{\text {ns }}$ & $0,09^{\text {ns }}$ & $2,47^{\text {ns }}$ & $0,00^{\text {ns }}$ \\
Y13 & $0,24^{\text {ns }}$ & $0,20^{\text {ns }}$ & $0,23^{\text {ns }}$ & $0,00^{\text {ns }}$ \\
Y14 & $0,35^{\mathrm{ns}}$ & $0,88^{\text {ns }}$ & $2,47^{\text {ns }}$ & $0,01^{\text {ns }}$ \\
\hline Tratamentos & $0,79^{\text {ns }}$ & $0,43^{\text {ns }}$ & $3,14^{\text {ns }}$ & 0,09 \\
Blocos & 0,03 & 2,57 & 1,21 & 0,37 \\
Resíduo & 0,67 & 0,96 & 5,83 & 4,29 \\
\hline C.V. $(\%)$ & 2,79 & 1,16 & 8,71 & \\
\hline
\end{tabular}

ns Não-significativo. *Significativo a $5 \%$ de probabilidade pelo teste F.

Tabela 8. Médias de comprimento da fibra, uniformidade, resistência e micronaire.

\begin{tabular}{lcccc}
\hline Tratamento & Comprimento $(2,5 \%-\mathrm{mm})$ & Uniformidade $(\%)$ & Resistência $(\mathrm{g} /$ tex $)$ & Micronaire $(\mu \mathrm{g} /$ in $)$ \\
\hline T1 & 29,48 & 84,02 & 27,45 & 4,35 \\
T2 & 29,40 & 84,67 & 26,80 & 4,52 \\
T3 & 30,25 & 84,78 & 28,10 & 4,55 \\
T4 & 28,75 & 84,17 & 28,87 & 4,45 \\
T5 & 29,45 & 84,43 & 28,90 & 4,48 \\
T6 & 29,20 & 84,57 & 28,13 & 4,40 \\
T7 & 29,12 & 84,00 & 28,52 & 4,40 \\
T8 & 29,08 & 84,65 & 27,33 & 4,43 \\
T9 & 30,50 & 83,92 & 27,27 & 4,55 \\
T10 & 29,23 & 84,60 & 28,20 & 4,60 \\
T11 & 29,58 & 84,28 & 26,22 & 4,65 \\
T12 & 84,40 & 26,28 & 4,83 \\
T13 & 29,30 & 85,05 & 27,70 & 4,28 \\
T14 & 29,40 & 54,75 & 27,32 & 4,45 \\
T15 & 29,08 & 28,87 & 4,48 \\
\hline
\end{tabular}


Tabela 9. Médias de comprimento, uniformidade, resistência e micronaire da fibra em cada comparação estabelecida.

\begin{tabular}{lcccc}
\hline Contraste & Comprimento $(2,5 \%-\mathrm{mm})$ & Uniformidade $(\%)$ & Resistência $(\mathrm{g} /$ tex $)$ & Micronaire $(\mu \mathrm{g} /$ in $)$ \\
\hline Y1 & $29,48-29,41^{\text {ns }}$ & $84,02-84,49^{\text {ns }}$ & $27,45-27,75^{\text {ns }}$ & $4,35-4,50^{\text {ns }}$ \\
Y2 & $29,08-29,47^{\text {ns }}$ & $85,05-84,75^{\text {ns }}$ & $27,32-28,87^{\text {ns }}$ & $4,45-4,48^{\text {ns }}$ \\
Y3 & $29,23-29,40^{\text {ns }}$ & $83,92-84,40^{\text {ns }}$ & $28,20-27,70^{\text {ns }}$ & $4,60-4,28^{*}$ \\
Y4 & $29,52-29,30^{\text {ns }}$ & $84,60-84,28^{\text {ns }}$ & $26,28-27,70^{\text {ns }}$ & $4,65-4,83^{\text {ns }}$ \\
Y5 & $29,40-29,08^{\text {ns }}$ & $84,67-84,00^{\text {ns }}$ & $26,80-27,33^{\text {ns }}$ & $4,52-4,43^{\text {ns }}$ \\
Y6 & $29,40-30,25^{\text {ns }}$ & $84,67-84,78^{\text {ns }}$ & $26,80-28,10^{\text {ns }}$ & $4,52-4,55^{\text {ns }}$ \\
Y7 & $30,25-28,75^{*}$ & $84,78-84,17^{\text {ns }}$ & $28,10-28,87^{\text {ns }}$ & $4,55-4,45^{\text {ns }}$ \\
Y8 & $29,45-29,20^{\text {ns }}$ & $84,43-84,57^{\text {ns }}$ & $28,90-28,13^{\text {ns }}$ & $4,48-4,40^{\text {ns }}$ \\
Y9 & $29,12-29,08^{\text {ns }}$ & $84,65-84,00^{\text {ns }}$ & $28,52-27,33^{\text {ns }}$ & $4,40-4,43^{\text {ns }}$ \\
Y10 & $29,08-30,50^{\text {ns }}$ & $84,00-84,65^{\text {ns }}$ & $27,33-27,27^{\text {ns }}$ & $4,43-4,55^{\text {ns }}$ \\
Y11 & $29,23-29,58^{\text {ns }}$ & $83,92-84,60^{\text {ns }}$ & $28,20-26,22^{\text {ns }}$ & $4,60-4,65^{\text {ns }}$ \\
Y12 & $29,40-29,16^{\text {ns }}$ & $84,67-84,48^{\text {ns }}$ & $26,80-27,76^{\text {ns }}$ & $4,52-4,52^{\text {ns }}$ \\
Y13 & $29,56-29,38^{\text {ns }}$ & $84,41-84,68^{\text {ns }}$ & $27,91-28,07^{\text {ns }}$ & $4,46-4,48^{\text {ns }}$ \\
Y14 & $29,08-29,44^{\text {ns }}$ & $84,00-84,57^{\text {ns }}$ & $27,33-28,28^{\text {ns }}$ & $4,43-4,38^{\text {ns }}$ \\
\hline
\end{tabular}

ns Não-significativo. *Significativo a $5 \%$ de probabilidade pelo teste $\mathrm{F}$.

os tratamentos, de acordo com Santana \& Wanderley (1995), as fibras são classificadas como médias (Tabela 8). Carvalho et al. (1994), comparando o efeito destes produtos aplicados em uma única vez entre 60 e 70 DAE, não encontraram efeitos significativos.

\section{Conclusões}

1. O parcelamento dos reguladores de crescimento proporciona maior redução da altura das plantas.

2. A combinação de produtos não tem efeito na redução da altura das plantas.

3. O peso do capulho é maior com a aplicação de regulador de crescimento.

4. A porcentagem de fibras não é influenciada pela aplicação de reguladores de crescimento.

5. A produtividade de fibras é influenciada pelo regulador e pelo esquema de parcelamento.

6. O comprimento das fibras e o índice Micronaire dependem da seqüência de aplicação.

\section{Referências}

ATHAYDE, M. L. F; LAMAS, F. M. Aplicação seqüencial de cloreto de mepiquat em algodoeiro. Pesquisa Agropecuária Brasileira, Brasília, v. 34, n. 3, p. 369-375, mar. 1999.

BANZATTO, D. A.; KRONKA, S. N. Experimentação agrícola. 2. ed. Jaboticabal : FUNEP, 1992. 247 p.

BELTRÃO, N. E. M. Uso de herbicidas, desfolhantes e hormônios no algodoeiro. In: SEMINÁRIO ESTADUAL
COM A CULTURA DO ALGODÃO EM MATO GROSSO, 3. , 1996, Cuiabá. Anais... Cuiabá : EMPAER-MT, [1996?]. p. 85-101. (EMPAER-MT. Documentos, 21).

CARVAlHO, L. H.; CHIAVEGATO, E. J.; CIA, E.; KONDO, J. I.; SABINO, J. C.; PETTINELLI JÚNIOR, A.; BORTOLETTO, N.; GALLO, P. B. Fitorreguladores de crescimento e capação na cultura algodoeira. Bragantia, Campinas, v. 53, n. 2, p. 247-254, 1994.

COTHREN, J. T.; OOSTERHUIS, D. M. Physiological impact of plant growth regulators in cotton. In: BELTWIDE COTTON PRODUCTION RESEARCH CONFERENCE, 1993, Dallas. Proceedings... Memphis : National Cotton Council, 1993. p. 128-132.

FURLANI JÚNIOR, E.; SILVA, N. M. da; FUZATTO, M. G.; CARVALHO, L. H.; CIA, E. Modos de aplicação de regulador de crescimento e níveis de adubação nitrogenada para o cultivar de algodão (Gossypium hirsutum L.) IAC 22, em diferentes densidades populacionais. In: CONGRESSO BRASILEIRO DE ALGODÃO, 2., 1999, Ribeirão Preto. Anais... Campina Grande : Embrapa-CNPA, 1999. p. 388-390.

HODGES, H. F.; REDDY, V. R.; REDDY, K. R. Mepiquat chloride and temperature effects on photosynthesis and respiration of fruiting cotton. Crop Science, Madison, v. 31, n. 5, p. 1302-1308, Sept./Oct. 1991.

LACA-BUENDIA, J. P. Efeito de doses de reguladores de crescimento no algodoeiro (Gossypium hirsutum L.). Revista Brasileira de Fisiologia Vegetal, Brasília, v. 1, n. 1, p. 109-113, 1989.

LAMAS, F. M. Cloreto de mepiquat, thidiazuron e ethephon aplicados no algodoeiro (Gossypium hirsutum), 
Ponta Porã-MS. Jaboticabal : UNESP, 1997. 192 p. Tese de Doutorado.

LAMAS, F. M.; STAUT, L. A. Adubação nitrogenada em cobertura e regulador de crescimento na cultura do algodoeiro em sistema plantio direto. Dourados : EmbrapaCPAO, 1999. 5 p. (Embrapa-CPAO. Comunicado Técnico, 5).

McCARTY, J. C.; HEDIN, P. A. Effects of 1,1dimethylpiperidinium chloride on the yields, agronomic traits, and alelochemicals of cotton (Gossypium hirsutum L.), a nine year study. Journal of Agricultural and Food Chemistry, Washington, v. 42, n. 10, p. 23022304, 1994.

MARUR, C. J. Fotossíntese e translocação de carboidratos em algodoeiros submetidos a déficit hídrico após aplicação de cloreto de mepiquat. Revista Brasileira de Fisiologia Vegetal, Brasília, v. 10, n. 1, p. 59-64, jan./abr. 1998.

PÍPOLO, A. E.; ATHAYDE, M. L. F.; PÍPOLO, V. C.; PARDUCCI, S. Comparação entre diferentes doses de cloreto de clorocolina, aplicadas em algodoeiro herbáceo.
Pesquisa Agropecuária Brasileira, Brasília, v. 28, n. 8, p. 915-923, ago. 1993.

REDDY, K. R.; BOONE, M. L.; REDDY, A. R.; HODGES, H. F.; TURNER, S. B.; McKINION, J. M. Developing and validating a model for plant growth regulator. Agronomy Journal, Madison, v. 87, n. 6, p. 1100-1105, Nov./Dec. 1995.

REDDY, V. R.; TRENT, A.; ACOCK, B. Mepiquat chloride and irrigation versus cotton growth and development. Agronomy Journal, Madison, v. 84, n. 6, p. 930-933, Nov./Dec. 1992.

SANTANA, J. C. F. de; WANDERLEY, M. J. R. Interpretação de resultados de análises de fibras, efetuadas pelo instrumento de alto volume (HVI) e pelo finurímetromaturímetro (FTM2). Campina Grande : EmbrapaCNPA,1995. 9 p. (Embrapa -CNPA. Comunicado Técnico, 41).

WALLACE, T. P.; SNIPES, C. E.; WHITE, B. W. Effect of single and multiple applications of mepiquat chloride on Mississipi cotton. Mississippi State: Mississippi State University, 1993. 5 p. (Mississippi Agricultural and Forest Experiment Station Research Report, 4). 